\title{
The first X-ray survey of Galactic luminous blue variables ${ }^{\star}$
}

\author{
Y. Nazé, ${ }^{\star \star}$, G. Rauw, and D. Hutsemékers' ${ }^{\star \star \star}$ \\ GAPHE, Département AGO, Université de Liège, Allée du 6 Août 17, Bât. B5C, 4000-Liège, Belgium \\ e-mail: naze@astro.ulg.ac. be
}

Received 7 September 2011 / Accepted 23 November 2011

\begin{abstract}
Aims. The X-ray emission of massive stars has been studied when these objects are in their main-sequence phase, as well as when they are in their Wolf-Rayet phase. However, the X-ray properties of the transitional luminous blue variable (LBV) phase remain unknown.

Methods. Using a dedicated but limited XMM-Newton survey and archival XMM-Newton and Chandra observations, we performed the first X-ray survey of LBVs: about half of the known LBVs or candidate LBVs were studied.

Results. Apart from the well known X-ray sources eta Car and Cyg OB2\#12, four additional LBVs are detected in this survey, though some doubt remains on the association with the X-ray source for two of these. For the other LBVs, upper limits on the flux were derived, down to $\log \left[L_{\mathrm{X}} / L_{\mathrm{BOL}}\right]-9.4$ for P Cyg. This variety in the strength of the X-ray emission is discussed, with particular emphasis on the potential influence of binarity.
\end{abstract}

Key words. X-rays: stars - stars : mass-loss - stars: early-type - stars: variables: S Doradus

\section{Introduction}

Luminous blue variables (LBVs), also called S-Doradus variables, form a class of peculiar massive stars mostly found in the top-left part of the Hertzsprung-Russell diagram (for a review, see Humphreys \& Davidson 1994). Their main characteristics are a high mass-loss rate (up to $10^{-4} M_{\odot} \mathrm{yr}^{-1}$ ), a high luminosity $\left(\sim 10^{6} L_{\odot}\right)$, and significant spectroscopic as well as photometric variability. These variations occur on several timescales and amplitudes, from common small-scale changes of about 0.1 mag to rare giant eruptions with $2.5 \mathrm{mag}$ variation.

The most emblematic objects in this category are those that have undergone unexpected, enormous variations (PCyg in the 17 th century, eta Car in the 19th century, and HD 5980 in 1993-5 and 1960-5). However, these dramatic cases should not be considered as the best representative objects of the class (Davidson 1989).

Luminous blue variables are often surrounded by young and massive circumstellar nebulae, which result from strong massloss episodes (see reviews by Lamers 1989; Smith 2011).

In view of their variability and the presence of nitrogenenriched circumstellar nebulae, LBVs are thought to be associated to a short $\left(<10^{5} \mathrm{yr}\right)$ unstable stage in the life of massive stars, between the main-sequence phase and the late Wolf-Rayet phase (e.g. Lamers et al. 2001). Their crucial role in the massloss process of massive stars has been revived in the last few years because the mass-loss rates of $\mathrm{O}$ and WR stars were revised downwards (e.g. Smith 2008).

While dedicated campaigns of X-ray observations have targeted massive stars in their O-star phase as well as in their

* Based on observations collected with XMM-Newton, an ESA Science Mission with instruments and contributions directly funded by ESA Member States and the USA (NASA), and with Chandra.

$\star \star$ Research Associate FRS-FNRS.

$\star \star \star$ Senior Research Associate FRS-FNRS.
Wolf-Rayet phase, not much was done for studying LBVs. Only the X-ray bright sources eta Car (Seward et al. 1979; Corcoran et al. 2010), Cyg OB2 \#12 (Harnden et al. 1979; Rauw 2011) and HD 5980 (Nazé et al. 2002, 2004, 2007), which display X-ray luminosities of $10^{34 . .35} \mathrm{erg} \mathrm{s}^{-1}$, have received attention. The sole exception may be PCyg, whose ROSAT HRI data are discussed in length by Berghöfer \& Wendker (2000) and are mentioned by Oskinova (2005a). This observation led to an upper limit on the luminosity of $\sim 10^{31} \mathrm{erg} \mathrm{s}^{-1}$. Finally, non-detections or a very weak detection of a few candidate LBVs are sometimes reported when discussing the X-ray emission of their home clusters, but without much detail: Pistol Star (Muno et al. 2006; Oskinova 2005b), Sher 25 (Moffat et al. 2002) and W243 (Clark et al. 2008).

This paper aims at bridging the gap between X-ray studies of $\mathrm{O}$ and WR stars by using the most sensitive $\mathrm{X}$-ray observatories available at the present time, XMM-Newton and Chandra. Using a dedicated (though limited) survey and archival data, we investigated the X-ray emission of about half of the known LBVs. The paper is organized as follows: Sect. 2 presents the LBV catalog used for our survey, Sect. 3 shows the available X-ray observations and their reduction, Sect. 4 lists our results, Sect. 5 discusses them and Sect. 6 finally summarizes the new information and concludes.

\section{The sample}

Two main S-Dor or LBV catalogs are available in the literature: van Genderen (2001) and Clark et al. (2005b). The latter lists more sources - 12 confirmed and 23 candidates (which we call cLBVs in the following) - and includes the 21 objects from van Genderen, with the exception of IRC+10420 and HD 148937 (which is of the peculiar Of?p type, see Walborn 1972 for the initial definition of this class and Nazé et al. 2011b, for an indepth study of this object in particular). 
Since Clark et al.'s work, two new cLBVs have been reported in the literature: MWC930 (Miroshnichenko et al. 2005) and 2MASS J17460562-2851319 (also known as G0.120-0.048, Mauerhan et al. 2010). Both were added to our catalog.

Recently, Spitzer surveys of the Galactic plane (e.g. MIPSGAL) have led to the discovery of many ring nebulae surrounding luminous central stars (Wachter et al. 2010; Gvaramadze et al. 2010b) and follow-up observations were conducted to determine the nature of these objects.

Wachter et al. (2010) list four known LBVs (or candidates) and six additional candidate LBVs. They also have six Be candidates for which they mention that their separation from the cLBVs is arbitrary and that these objects should therefore be considered as potential LBVs, too. Gvaramadze et al. (2010b) reported the detection of two additional cLBVs and a (known, though not included in the Clark et al. compilation) cLBV. Note that one of these, MN112, was identified as Be in Wachter et al.'s list (under the name 2MASS J19443759+2419058).

Expanding on these works, Wachter et al. (2011) list eight additional LBV candidates (one that was classified as Be in her previous paper) and four additional Be candidates (two already classified as such in their previous paper). They also mention finding six additional LBV candidates and one Be candidate from the follow-up observations of the sample of Gvaramadze et al. (2010b).

Considering these new detections, we sum up Clark's list of 35 LBVs or cLBVs, 2 recent additions, 21 objects from Wachter's sample and 9 from Gvaramadze's sample. This enlarges the (c)LBV catalog to 67 objects. They are given in Table 1, along with their main properties, when known (see quoted references). Typical errors on $\log (T)$ and $\log (L)$ are 0.05 and 0.2 dex, respectively (van Genderen 2001). More generally, it must be kept in mind that the properties of LBVs are much more uncertain than those of $\mathrm{O}$ and WR stars. Names and positions come from the Simbad database and Gvaramadze et al. (2010b) for their sample - RA and Dec were rounded to two decimal digits. For GRS 79.29+0.46, the Simbad and van Genderen coordinates do not agree well, and a revised estimate of the position was found in Jiménez-Esteban et al. (2010): the coordinates quoted in Table 1 correspond to those of 2MASS J20314228+4021591, the (bright) 2MASS source closest to the Simbad position as well as to the van Genderen and Jiménez-Esteban coordinates.

\section{X-ray observations}

We have obtained XMM observations for a limited LBV survey (ObsID = 0600030, PI Nazé). To enlarge the sample as much as possible, we also searched the archives for additional, serendipitous observations of the known LBVs or cLBVs lying within 15' (for XMM-Newton) or 10' (for Chandra) of the on-axis position. Only imaging exposures (i.e. no XMM "timing" mode or Chandra "continuous clocking" mode) longer than $5 \mathrm{ks}$ were considered. Indeed, the quality of these serendipitous observations is often not as good as for the requested on-axis data, because the source usually lies far off-axis and the effective exposure time may not be very long.

In total, including our targets, XMM-Newton has observed 26 (c)LBVs while Chandra has observed 21 objects. A closer look indicates that 16 targets are in common, implying that 31 (c)LBVs, or half of the catalog, have been observed in X-rays (see crosses in the last columns of Table 1). Note that if we restrict ourselves to the Clark catalog, XMM-Newton has observed
19 objects and Chandra 16, with 13 sources in common: X-ray observations therefore exist for two-thirds of Clark's catalog.

Two final remarks must be made. First, the datasets for the two well-known X-ray emitters eta Car and Cyg OB2 \#12 have not been re-reduced or re-analyzed, because many published papers already discuss them in detail (see Sects. 4.1.1 and 4.1.3). Second, the analysis of the ten sources close to the Galactic center relies on the Chandra data only, because XMM-Newton is not very good at separating sources in this crowded region.

\subsection{XMM-Newton}

The XMM-Newton EPIC data were reduced in a standard way using SAS v10.0. After the pipeline processing (tasks emproc, epproc), the recommended pattern filtering was applied (PATTERN $\leq 12$ and XMMEA_EM filter for MOS, PATTERN $\leq 4$ and FLAG $=0$ for $\mathrm{pn}$ ). The event files were additionally filtered to reject times affected by background flares. Images in the $0.5-8.0 \mathrm{keV}$ domain were then produced and used for the detection algorithm edetectchain when only one exposure was available ${ }^{1}$. The logarithmic likelihood for detection was set at 10 , equivalent to a $4 \sigma$ detection. Sensitivity maps for likelihoods $\ln L=2.3$ (to be consistent with the Galactic center data, see Sect. 3.4) and combining all instruments were also produced using the task esensmap. These maps provide equivalent on-axis limiting count rates, which were transformed into actual fluxes using WebPimms ${ }^{2}$. The chosen model is a simple optically-thin thermal emission with a temperature of $0.6 \mathrm{keV}$ and an absorption directly related to the known extinctions of the targets (using $N_{\mathrm{H}}=5.8 \times 10^{21} \times E(B-V) \mathrm{cm}^{-2}$, Bohlin et al. 1978). Using temperatures of 0.3 or $1.0 \mathrm{keV}$ does not change the results by much: for an observed flux of $10^{-15} \mathrm{erg} \mathrm{cm}^{-2} \mathrm{~s}^{-1}$ and a representative absorbing column of $10^{22} \mathrm{~cm}^{-2}$, the total EPIC count rates vary by $10 \%$ in these cases compared to the $0.6 \mathrm{keV}$ case. Because our limits are certainly not more accurate than $10 \%$, it means that they are quite insensitive to the adopted temperature. For the Wachter et al. or Gvaramadze et al. sources, no information on distance, bolometric luminosity or reddening is available. To obtain a limit in (observed) flux, we assumed a representative absorbing column of $10^{22} \mathrm{~cm}^{-2}$. Changing the latter by an order of magnitude (i.e. $10^{21}$ or $10^{23} \mathrm{~cm}^{-2}$ ) would change the total EPIC count rate for a given observed flux by a factor of $\sim 2$, whatever the filter. We therefore consider that the flux limits for these objects are accurate within a factor of 2 .

For Chandra data, the upper limit determination is slightly different (see next section), but we checked on the XMM-Newton data of AG Car that both methods provide similar estimates, and therefore are fully compatible with each other.

When several exposures of a given region exist, a recent task added to the SAS, emosaicproc, is supposed to help combining them. However, its use is not straightforward. For 2MASS J18022233-2238002, two of the three exposures had a strange bug in the attitude file that prevents their use with this task (see also below, in the discussion of this source). For W243, the start time of the Rev 1499 summary file was wrong and had to be corrected by hand before the task could work. In addition, while the detection algorithm ran otherwise smoothly, the creation of a sensitivity map combining all exposures and all instruments is not always possible, because the task esensmap only accepts combinations of $<10$ input files. However, the effective area is

\footnotetext{
1 See the analysis threads for detail on its use: http://xmm.esac. esa.int/sas/current/documentation/threads/

2 http://heasarc.gsfc.nasa.gov/Tools/w3pimms.html
} 
Table 1. Catalog of the known LBVs and LBV candidates (see Sect. 2).

\begin{tabular}{|c|c|c|c|c|c|c|c|c|c|}
\hline Simbad name & $\begin{array}{l}\text { RA(J2000) } \\
\text { hh mm ss }\end{array}$ & $\begin{array}{c}\operatorname{Dec}(\mathrm{J} 2000) \\
\circ,{ }^{\prime}\end{array}$ & $\begin{array}{c}\log \left(T_{\text {eff }}\right) \\
\mathrm{K}\end{array}$ & $\begin{array}{c}\log \left(L_{\mathrm{BOL}}\right) \\
L_{\odot} \\
\end{array}$ & $E(B-V)$ & $\begin{array}{c}d \\
\mathrm{kpc}\end{array}$ & Ref. $^{a}$ & $\mathrm{X} ?$ & $\mathrm{C}$ ? \\
\hline \multicolumn{10}{|l|}{ Clark's catalog } \\
\hline \multicolumn{10}{|l|}{ A. Confirmed LBVs } \\
\hline eta Car & 104503.59 & -594104.26 & $4.36-4.18$ & $5.14-6.34$ & 0.50 & 2.3 & vG01 & $\mathrm{x}$ & $\mathrm{x}$ \\
\hline P Cyg & 201747.20 & +380158.55 & 4.26 & 5.70 & 0.51 & 1.7 & vG01 & $\mathrm{x}$ & \\
\hline HD 168607 & 182114.89 & -162231.76 & 3.97 & 5.38 & 1.55 & 2.2 & vG01 & & \\
\hline $\mathrm{AG}$ Car & 105611.58 & -602712.81 & $4.46-4.13$ & $6.14-6.22$ & 0.63 & 6.0 & vG01 & $\mathrm{x}$ & \\
\hline HR Car & 102253.84 & -593728.38 & $4.34-3.90$ & $5.90-5.62$ & 1.00 & 5.2 & vG01 & & \\
\hline HD 160529 & 174159.03 & -333013.71 & 3.95 & 5.46 & 1.10 & 2.5 & vG01 & $\mathrm{x}$ & \\
\hline WRAY 15-751 & 110840.06 & -604251.7 & 4.48 & 5.91 & 1.60 & 6.0 & pa06,vG01 & & \\
\hline qF 362 & 174617.98 & -284903.46 & 4.05 & 6.25 & 8.00 & 11.5 & na09 & $\mathrm{x}$ & $\mathrm{x}$ \\
\hline AFGL 2298 & 190010.89 & +034547.1 & $4.01-4.07$ & $6.11-6.30$ & 9.00 & 10.0 & $\mathrm{cl09b}$ & & \\
\hline GAL $024.73+00.69$ & 183355.29 & -065838.7 & 4.08 & 5.6 & 3.40 & 5.2 & cl03 & $\mathrm{x}$ & \\
\hline $\mathrm{Cl}^{*}$ Westerlund $1 \mathrm{~W} 243$ & 164707.50 & -455229.16 & 3.93 & 5.86 & 4.35 & 4.5 & ri09,cl05 & $\mathrm{x}$ & $\mathrm{x}$ \\
\hline GCIRS 34W & 174539.73 & -290026.50 & 4.29 & 5.5 & 10.80 & 7.6 & $\mathrm{ma07}$ & $\mathrm{x}$ & $\mathrm{x}$ \\
\hline \multicolumn{10}{|l|}{ B. Candidate LBVs } \\
\hline NAME VI Cyg 12 & 203240.96 & +411429.28 & 4.11 & 6.42 & 3.40 & 1.7 & vG01 & $\mathrm{x}$ & $\mathrm{x}$ \\
\hline GRS $79.29+0.46$ & 203142.28 & +402159.13 & 4.40 & 6.30 & 5.00 & 2.0 & vG01 & & $\mathrm{x}^{b}$ \\
\hline Pistol Star & 174615.24 & -285003.58 & 4.07 & 6.20 & 8.00 & 11.5 & na09 & $\mathrm{x}$ & $\mathrm{x}$ \\
\hline HD 168625 & 182119.55 & -162226.06 & 4.08 & 5.34 & 1.50 & 2.2 & vG92 & & \\
\hline zet01 Sco & 165359.73 & -422143.31 & 4.26 & 6.10 & 0.66 & 2.0 & cr06 & & \\
\hline HD 326823 & 170653.91 & -423639.74 & 4.34 & 5.30 & 1.15 & 2.0 & mr07 & & \\
\hline HD 316285 & 174814.04 & -280053.13 & 4.18 & 5.44 & 1.81 & 1.9 & vG01 & $\mathrm{x}$ & \\
\hline Hen 3-519 & 105359.59 & -602644.31 & 4.48 & 6.26 & 1.30 & 8.0 & vG01 & & \\
\hline HD 80077 & 091554.79 & -495824.58 & 4.23 & 6.30 & 1.47 & 3.0 & vG01 & & \\
\hline AS 314 & 183926.11 & -135047.19 & 4.01 & 4.90 & 0.90 & 8.0 & vG01 & & \\
\hline MWC 314 & 192133.97 & +145256.89 & 4.48 & 6.34 & 1.84 & 3.0 & vG01 & & \\
\hline GRS $25.5+0.2$ & 183705.21 & -062938.0 & & & 10.00 & 14.5 & vG01 & $\mathrm{x}$ & \\
\hline GAL $026.47+00.02$ & 183932.24 & -054420.5 & 4.23 & 6.0 & 3.80 & 6.5 & $\mathrm{cl03}$ & & $\mathrm{x}$ \\
\hline WRAY 17-96 & 174135.45 & -300638.8 & 4.11 & 6.26 & 2.84 & 4.5 & eg02 & & \\
\hline WR102ka & 174618.12 & -290136.6 & 4.40 & 6.5 & 8.0 & 8.0 & ba08 & $\mathrm{x}$ & $\mathrm{x}$ \\
\hline NAME LBV 1806-20 & 180840.31 & -202441.1 & & 6.3 & 9.4 & 11.8 & fi04,ei04 & $\mathrm{x}$ & $\mathrm{x}$ \\
\hline NAME Sher 25 star & 111507.8 & -611517 & 4.35 & 5.9 & 1.60 & 6.3 & sm02 & & $\mathrm{x}$ \\
\hline [OMN2000] LS1 & 192347.64 & +143638.4 & $4.12-4.14$ & 5.75 & 3.50 & 6.0 & cl09a & & $\mathrm{x}$ \\
\hline GCIRS 16NE & 174540.26 & -290027.09 & 4.29 & 5.9 & 8.30 & 7.6 & ma07 & $\mathrm{x}$ & $\mathrm{x}$ \\
\hline GCIRS 16C & 174540.13 & -290027.64 & 4.24 & 5.9 & 8.30 & 7.6 & $\mathrm{ma07}$ & $\mathrm{x}$ & $\mathrm{x}$ \\
\hline GCIRS 16SW & 174540.12 & -290029.06 & & & & & & $\mathrm{x}$ & $\mathrm{x}$ \\
\hline GCIRS 16NW & 174540.05 & -290026.87 & & & & & & $\mathrm{x}$ & $\mathrm{x}$ \\
\hline GCIRS 33SE & 174540.02 & -290031.0 & 4.26 & 5.75 & 9.00 & 7.6 & ma07 & $\mathrm{x}$ & $\mathrm{x}$ \\
\hline \multicolumn{10}{|l|}{ New additions (cLBVS) } \\
\hline 2MASS J17460562-2851319 & 174605.625 & -285131.92 & & $6.2-6.6$ & 8.00 & 11.5 & mu10 & $\mathrm{x}$ & $\mathrm{x}$ \\
\hline MWC 930 & 182625.24 & -071317.8 & 4.34 & 5.5 & 2.5 & 3.5 & $\mathrm{mi05}$ & $\mathrm{x}$ & \\
\hline \multicolumn{10}{|l|}{ Wachter's sources (cLBVs) } \\
\hline 2MASS J15484207-5507422 & 154842.07 & -550742.21 & & & & & & & \\
\hline 2MASS J16290377-4746264 & 162903.78 & -474626.48 & & & & & & & \\
\hline 2MASS J16364278-4656207 & 163642.78 & -465620.73 & & & & & & & $\mathrm{x}^{b}$ \\
\hline 2MASS J17082913-3925076 & 170829.14 & -392507.68 & & & & & & & \\
\hline 2MASS J17110094-3945174 & 171100.94 & -394517.45 & & & & & & $\mathrm{x}$ & \\
\hline 2MASS J18415965-0515409 & 184159.65 & -051540.93 & & & & & & & $\mathrm{x}$ \\
\hline 2MASS J16431636-4600424 & 164316.37 & -460042.42 & & & & & & & \\
\hline 2MASS J16493770-4535592 & 164937.70 & -453559.27 & & & & & & & \\
\hline 2MASS J17435981-3028384 & 174359.85 & -302838.5 & & & & & & & \\
\hline 2MASS J18133121-1856431 & 181331.21 & -185643.20 & & & & & & & \\
\hline 2MASS J19325284+1742303 & 193252.85 & +174230.33 & & & & & & & \\
\hline 2 MASS J19443759+2419058 & 194437.60 & +241905.87 & & & & & gv10 & & \\
\hline 2MASS J16313781-4814553 & 163137.82 & -481455.30 & & & & & & & \\
\hline 2MASS J16461734-4508478 & 164617.35 & -450847.85 & & & & & & $\mathrm{x}$ & \\
\hline 2MASS J17374754-3137333 ${ }^{d}$ & 173747.54 & -313733.38 & & & & & & & \\
\hline 2MASS J17374730-3137370 ${ }^{d}$ & 173747.31 & -313737.07 & & & & & & & \\
\hline 2MASS J17421401-2955360 & 174214.02 & -295536.06 & & & & & & & \\
\hline 2MASS J18022233-2238002 & 180222.34 & -223800.24 & & & & & & $\mathrm{x}$ & \\
\hline 2MASS J18455593-0308297 & 184555.94 & -030829.72 & & & & & & & \\
\hline
\end{tabular}


Table 1. continued.

\begin{tabular}{|c|c|c|c|c|c|c|c|c|c|}
\hline Simbad name & $\begin{array}{l}\text { RA(J2000) } \\
\text { hh mm ss }\end{array}$ & $\begin{array}{c}\operatorname{Dec}(\mathrm{J} 2000) \\
0_{\prime \prime \prime \prime}\end{array}$ & $\underset{\mathrm{K}}{\log \left(T_{\text {eff }}\right)}$ & $\begin{array}{c}\log \left(L_{\mathrm{BOL}}\right) \\
L_{\odot}\end{array}$ & $E(B-V)$ & $\begin{array}{c}d \\
\mathrm{kpc}\end{array}$ & $\operatorname{Ref}^{a}$ & $\mathrm{X}$ ? & $\mathrm{C}$ ? \\
\hline 2MASS J18510295-0058242 & 185102.95 & -005824.21 & & & & & & & \\
\hline 2MASS J18070516-2015163 & 180705.17 & -201516.31 & & & & & & & \\
\hline 2MASS J19011669+0355108 & 190116.69 & +035510.81 & & & & & & & \\
\hline \multicolumn{10}{|l|}{ Gvaramadze's sources (cLBVs) } \\
\hline MN13 & 134233.08 & -624811.3 & & & & & & & \\
\hline MN39 & 161026.55 & -512125.3 & & & & & & & \\
\hline MN41 & 162634.28 & -502101.9 & & & & & & & \\
\hline MN53 & 170924.78 & -400845.6 & & & & & & $\mathrm{x}$ & $\mathrm{x}$ \\
\hline MN79 & 182833.41 & -114644.2 & & & & & & $\mathrm{x}$ & $\mathrm{x}$ \\
\hline MN101 & 190624.54 & 082201.6 & & & & & & & \\
\hline MN107 & 192403.34 & 133949.4 & & & & & & & \\
\hline MN46 & 164316.37 & -460042.4 & & & & & & & \\
\hline MN83 & 183923.01 & -055319.9 & & & & & & & $\mathrm{x}$ \\
\hline
\end{tabular}

Notes. X and C refer to XMM-Newton and Chandra observations, respectively. ${ }^{(b)}$ Not yet publicly available. ${ }^{(c)}$ In Gvaramadze et al. (2010b), this source appears as MN112. ${ }^{(d)}$ Wachter et al. (2011) quote as LBV candidate their source \#27 of Wachter et al. (2010). However, in the latter paper, there are two \#27 sources, a and b. Both are thus quoted together here.

References. ${ }^{(a)}$ ba08 = Barniske et al. (2008), c103 = Clark et al. (2003), cl05 = Clark et al. (2005a), cl09a $=$ Clark et al. $(2009 \mathrm{a})$, cl09b $=$ Clark et al. (2009b), cr06 = Crowther et al. (2006), eg02 = Egan et al. $(2002)$, ei04 = Eikenberry et al. $(2004)$, fi04 = Figer et al. $(2004)$, gv10 = Gvaramadze et al. (2010a), ma07 = Martins et al. (2007), mr07 = Marcolino et al. (2007), mu10 = Mauerhan et al. (2010), mi05 = Miroshnichenko et al. (2005), na09 = Najarro et al. (2009), pa06 = Pasquali et al. (2006), ri09 = Ritchie et al. (2009), sm02 = Smartt et al. $(2002)$, vG92 = van Genderen et al. (1992), vG01 = van Genderen (2001).

not always the dominant factor limiting the sensitivity of the $X M M-N e w t o n$ data (see Sects. 4.1.2 and 4.3.10) and the impact of this problem is thus quite limited.

Table 2 lists the results of our analysis of the XMMNewton data.

Finally, a check was made using the XMM-Newton upper limit server ${ }^{3}$. In three cases, only slew data were used, which led to very high upper limits compared to data from pointed observations, as could be expected. In addition, several detections with varying count rates were reported for W243 as well as a detection for P Cyg, but they are due to source confusion in these regions (see details on these sources below). In all cases except for those just discussed, the server finds upper limits that are compatible with ours, though slightly higher.

\subsection{Chandra}

The pipeline data (level 2) available in the Chandra archives were downloaded but not re-processed. Additional work was done with CIAO 4.2 and CALDB 4.3.1. We first defined the source and background regions. The source regions are circular, with radii of $5^{\prime \prime}$ (i.e. $10 \mathrm{px}$ ), while the background regions were chosen as surrounding annuli of radii $5^{\prime \prime}$ and $15^{\prime \prime}$. The sources W243 and LBV 1806-20 have close X-ray companions, so that the annular background regions were replaced by nearby circular regions of $5^{\prime \prime}$ radii in these two cases. We then used the task specextract to obtain unbinned spectra of source and background regions, as well as their corresponding response matrices. When several observations were available, the task combine_spectra was used to co-add the spectra.

If the source was detected, the flux in the $0.5-8.0 \mathrm{keV}$ band was then readily estimated using an absorbed optically-thin thermal model, with temperature fixed to $0.6 \mathrm{keV}$ whenever less than $100 \mathrm{cts}$ were recorded and free otherwise, and the absorptions were always fixed to their known values (see previous section). In case of non-detection, the count numbers in the two

\footnotetext{
${ }^{3}$ http://xmm.esac.esa.int/UpperLimitsServer/
}

regions, their areas, and the exposure times provide upper limits with $90 \%$ significance using the task aprates ${ }^{4}$, which relies on Bayesian statistics to evaluate errors. Using the calculated response matrices and a "fakeit" run under Xspec, we then transformed these count rates into actual fluxes assuming the same model as above.

In two cases, the available data were taken using HRC rather than ACIS, implying that no spectral information is available. For LBV 1806-20, two other ACIS datasets are available. We therefore only checked that the limit derived in the same regions using the task aprates on the HRC data was compatible with the ACIS results. For MN53, the HRC data are the sole available ones, and the HRC limit on the count rate found with aprates was transformed into actual flux using WebPIMMs and the above model.

Table 3 lists our results. As for XMM-Newton, we considered to have a secure detection when the source is detected with at least $4 \sigma$ significance.

Finally, a search was made for the cataloged (c)LBVs in the Chandra Source Catalog (CSC) ${ }^{5}$. X-ray sources were only found close to GAL 026.47+00.02, W243 and Sher 25. For the former, the X-ray source lies at $0.2^{\prime \prime}$, implying detection, but for the last two objects, the CSC X-ray sources lie at $>5^{\prime \prime}$, casting doubt on their identification with the LBVs.

\subsection{Proprietary data}

Sher 25 is a special case in our survey because five observations exist, but four of these were still proprietary at the time of the analysis. These additional data greatly enhance the detection limit, though, because the total exposure time is then multiplied by 10 . The PI of these data, L. Townsley, kindly made them

\footnotetext{
${ }^{4}$ See http://cxc.harvard.edu/ciao/threads/upperlimit/ We used it with alpha $=0.99$ (the PSF fraction encircled by the source region) and beta $=0.01$ (the PSF fraction encircled by the background region).

5 http://cxc.harvard.edu/csc/index.html
} 
Table 2. XMM-Newton imaging observations of (c)LBVs.

\begin{tabular}{|c|c|c|c|c|c|c|c|}
\hline Simbad name & Obsid & Rev. & $\begin{array}{c}\text { OFA } \\
(')\end{array}$ & $\begin{array}{c}\text { EPIC count rate } \\
\mathrm{ct} \mathrm{s}^{-1}\end{array}$ & $\begin{array}{c}F_{\mathrm{X}}^{\mathrm{obs}} \\
\mathrm{erg} \mathrm{cm}^{-2} \mathrm{~s}^{-1}\end{array}$ & $\begin{array}{c}L_{\mathrm{X}}^{\text {unabs }} \\
\mathrm{erg} \mathrm{s}^{-1}\end{array}$ & $\log \left[L_{\mathrm{X}} / L_{\mathrm{BOL}}\right]$ \\
\hline P Cyg & 0600030201 & 1891 & 0. & $<0.0010$ & $<1.0 \times 10^{-15}$ & $<8.3 \times 10^{29}$ & $<-9.4$ \\
\hline AG Car & 0600030101 & 1853 & 0. & $<0.0009$ & $<8.1 \times 10^{-16}$ & $<1.0 \times 10^{31}$ & $<-8.7 . .8 .8$ \\
\hline HD 160529 & 0600030701 & 1797 & 0. & $<0.0008$ & $<7.8 \times 10^{-16}$ & $<3.2 \times 10^{30}$ & $<-8.5$ \\
\hline GAL $024.73+00.69$ & 0301880301 & 1145 & 5.6 & $<0.006$ & $<8.4 \times 10^{-15}$ & $<7.2 \times 10^{32}$ & $<-6.3$ \\
\hline \multirow[t]{5}{*}{$\mathrm{Cl}^{*}$ Westerlund $1 \mathrm{~W} 243$} & 0410580601 & 1317 & 0.5 & $<0.0017$ & $<2.6 \times 10^{-15}$ & $<2.3 \times 10^{32}$ & $<-7.1$ \\
\hline & 0505290201 & 1409 & 0.5 & & & & \\
\hline & 0505290301 & 1499 & 0.5 & & & & \\
\hline & 0555350101 & 1593 & 0.5 & & & & \\
\hline & 0604380101 & 1778 & 0.5 & & & & \\
\hline HD 316285 & 0112970101 & 0145 & 7.8 & $<0.0013$ & $<1.4 \times 10^{-15}$ & $<6.1 \times 10^{30}$ & $<-8.2$ \\
\hline GRS $25.5+0.2$ & 0400910301 & 1256 & 10. & $<0.0025$ & $<5.7 \times 10^{-15}$ & $<1.7 \times 10^{34}$ & \\
\hline \multirow[t]{9}{*}{ NAME LBV $1806-20^{a}$} & 0205350101 & 0869 & 0. & $<0.01$ & $<2.2 \times 10^{-14}$ & $<4.1 \times 10^{34}$ & $<-5.3$ \\
\hline & 0164561101 & 0884 & 0. & & & & \\
\hline & 0164561401 & 1066 & 0. & & & & \\
\hline & 0406600301 & 1157 & 0. & & & & \\
\hline & 0406600401 & 1237 & 0. & & & & \\
\hline & 0502170301 & 1428 & 0. & & & & \\
\hline & 0502170401 & 1523 & 0. & & & & \\
\hline & 0554600301 & 1601 & 0. & & & & \\
\hline & 0554600401 & 1691 & 0 . & & & & \\
\hline $\mathrm{MCW} 930^{b}$ & 0650591501 & 2059 & 7.5 & $<0.0006$ & $<1.9 \times 10^{-15}$ & $<5.0 \times 10^{31}$ & $<-7.4$ \\
\hline 2MASS J17110094-3945174 & 0502080301 & 1431 & 0.9 & $<0.003$ & $<3.2 \times 10^{-15}$ & & \\
\hline 2MASS J16461734-4508478 & 0164561001 & 0873 & 2.6 & $<0.004$ & $<4.9 \times 10^{-15}$ & & \\
\hline 2MASS J18022233-2238002 & 0135742801 & 0600 & 11. & $<0.0035$ & $<3.8 \times 10^{-15}$ & & \\
\hline $\mathrm{MN}^{b} 3^{b}$ & 0148690101 & 0681 & 6.9 & $<0.0005$ & $<1.5 \times 10^{-15}$ & & \\
\hline MN79 & 0051940401 & 0229 & 3.7 & $<0.0012$ & $<1.3 \times 10^{-15}$ & & \\
\hline eta Car & \multirow{2}{*}{\multicolumn{4}{|c|}{ see Corcoran et al. (2010) }} & $0.06 . .3 \times 10^{-10}$ & & $\sim-5$ \\
\hline NAME VI Cyg 12 & & & & & $2.7 \times 10^{-12}$ & $8.2 \times 10^{33}$ & -6.1 \\
\hline
\end{tabular}

Notes. A null off-axis angle (OFA) indicates an on-axis observation. Column 1 provides the (c)LBV name, Cols. 2 to 4 the details of the observation(s) used, and the next columns list the derived EPIC count rates, fluxes, and luminosities in the $0.5-8.0 \mathrm{keV}$ energy range. Limits correspond to a 90\% chance of being detected if brighter. ${ }^{(a)}$ Three additional observations (Obsid - Rev =0148210101 - 0607, $0148210401-0701$, and $0164561301-0960)$ are available but are totally affected by strong flares: they were therefore discarded from the analysis. ${ }^{(b)}$ Refers to EPIC MOS data only.

available to us. The full dataset (the public exposure and the four private observations) was reduced and combined using the ACIS Extract software package ${ }^{6}$ by Broos: we used here an extraction specifically made at the position of Sher 25 on the full dataset.

Similarly, a deep analysis of the X-ray emission of MCW 930 was possible because the PI of the XMM-Newton data, L. Bassani, kindly made the observation available to us before the end of her proprietary period. These data were reduced in the same way as our other XMM-Newton data (see Sect. 3.1). Note that there is also a Chandra dataset $(400638$ - 7528) for this source, but it has an exposure time of only $5 \mathrm{ks}$; moreover, this cLBV appears on the edge of a CCD, prohibiting the derivation of any meaningful flux limit.

It may be worth noting that two additional sources (GRS 79.29+0.46 and 2MASS J16364278-4656207, see Table 1) have Chandra data, but they are not yet publicly available.

\subsection{The Galactic center survey}

Ten of the cataloged cLBVs lie close to the Galactic center. A survey of this region, using 2Ms of Chandra time, was reported

\footnotetext{
6 The ACIS Extract software package and User's Guide are available at http://www.astro.psu.edu/xray/acis/acis_analysis. html, see also Broos et al. (2010).
}

by Muno et al. (2009). This paper and its associated on-line resources ${ }^{7}$ provide source lists and source spectra, as well as sensitivity maps in the $0.5-8.0 \mathrm{keV}$ energy range (see their Fig. 9). We used the sensitivity map where sources brighter than the quoted limiting photon flux have $>90 \%$ chance of being detected (the other one available corresponds to a $>50 \%$ chance). This $90 \%$ probability corresponds to $1.65 \sigma$ or a logarithmic likelihood of 2.3 .

\section{Results}

In this section, we present the results of this survey. We begin by presenting the detections, and then continue with the doubtful detections and the non-detections. Whenever the X-ray emission of the object has been discussed in the past, it is clearly mentioned.

\subsection{Detections}

\subsection{1. eta Car}

Of all LBVs, eta Car is certainly the most spectacular, luminous, and best studied object... but not necessarily the one best

7 See http://www.srl.caltech.edu/gc_project/xray.html and tables on the journal webpage (CDS tables are incomplete and tables on the Caltech website contain errors). 
Table 3. Chandra imaging observations of (c)LBVs.

\begin{tabular}{|c|c|c|c|c|c|c|c|}
\hline Simbad name & Seqnum & Obs_ID & $\begin{array}{l}\text { OFA } \\
(\prime)\end{array}$ & $\begin{array}{c}\text { Count rate } \\
\mathrm{ct} \mathrm{s}^{-1}\end{array}$ & $\begin{array}{c}F_{\mathrm{X}}^{\mathrm{obs}} \\
\operatorname{erg~cm}^{-2} \mathrm{~s}^{-1}\end{array}$ & $\begin{array}{l}L_{X}^{\text {unabs }} \\
\mathrm{erg} \mathrm{s}^{-1}\end{array}$ & $\log \left[L_{\mathrm{X}} / L_{\mathrm{BOL}}\right]$ \\
\hline \multirow[t]{2}{*}{$\mathrm{Cl}^{*}$ West. $1 \mathrm{~W} 243$} & 200344 & 5411 & 1.7 & $0.00029 . .61 \pm 0.00015$ & $1.7 \ldots 3.5 \times 10^{-15}:$ & $1.5 \ldots 3.1 \times 10^{32}:$ & $-7.3 \ldots-7.0:$ \\
\hline & 200344 & 6283 & 2.1 & & & & \\
\hline GAL $026.47+00.02$ & 400603 & 7493 & 4.3 & $0.0161 \pm 0.0009$ & $(1.73 \pm 0.10) \times 10^{-13}$ & $(4.3 \pm 0.2) \times 10^{33}$ & $-5.95 \pm 0.02$ \\
\hline \multirow[t]{2}{*}{ NAME LBV $1806-20^{a}$} & 500599 & $6251 \mathrm{H}$ & 0.4 & $<0.0009$ & $<3.7 \times 10^{-14}$ & $<6.9 \times 10^{34}$ & $<-5.0$ \\
\hline & 500042 & 746 & 0.6 & $<0.0011$ & $<1.0 \times 10^{-14}$ & $<2.0 \times 10^{34}$ & $<-5.6$ \\
\hline \multirow[t]{5}{*}{ NAME Sher 25 star } & 200058 & 633 & 0.6 & $<0.000017$ & $<1.1 \times 10^{-16}$ & $<4.9 \times 10^{30}$ & $<-8.8$ \\
\hline & 200666 & 12328 & 0.6 & & & & \\
\hline & 200666 & 12329 & 0.5 & & & & \\
\hline & 200666 & 12330 & 0.5 & & & & \\
\hline & 200666 & 13162 & 0.5 & & & & \\
\hline \multirow[t]{2}{*}{ [OMN2000] LS1 } & 200132 & 2524 & 5.9 & $<0.00018$ & $<1.4 \times 10^{-15}$ & $<1.6 \times 10^{32}$ & $<-7.1$ \\
\hline & 200132 & 3711 & 5.9 & & & & \\
\hline $\begin{array}{c}\text { 2MASS J18415965 } \\
-0515409\end{array}$ & 400662 & 7552 & 6.2 & $<0.00016$ & $<1.3 \times 10^{-15}$ & & \\
\hline MN53 & 500140 & $1963 \mathrm{H}$ & 7.4 & $<0.002$ & $<2.4 \times 10^{-14}$ & & \\
\hline MN79 & 500629 & 6675 & 1.7 & $<0.00025$ & $<1.0 \times 10^{-15}$ & & \\
\hline${\mathrm{MN} 83^{b}}^{b}$ & 400603 & 7493 & 5.2 & $<0.0002$ & $<1.5 \times 10^{-15}$ & & \\
\hline \multicolumn{2}{|l|}{ eta Car } & \multirow{2}{*}{\multicolumn{3}{|c|}{$\begin{array}{l}\text { see Corcoran et al. (2010) } \\
\text { see Rauw (2011) }\end{array}$}} & $0.06 \ldots 3 \times 10^{-10}$ & & $\sim-5$ \\
\hline \multicolumn{2}{|l|}{ NAME VI Cyg 12} & & & & $2.7 \times 10^{-12}$ & $8.2 \times 10^{33}$ & -6.1 \\
\hline \multicolumn{2}{|l|}{ Galactic center } & \multicolumn{3}{|c|}{ photon fluxes $\left(\mathrm{ph} \mathrm{cm}^{-2} \mathrm{~s}^{-1}\right)$} & & & \\
\hline \multirow{2}{*}{\multicolumn{2}{|c|}{$\begin{array}{l}\text { qF } 362 \\
\text { GCIRS 34W }\end{array}$}} & & & $<1.5 \times 10^{-6}$ & $<5.6 \times 10^{-15}$ & $<7.8 \times 10^{33}$ & $<-5.9$ \\
\hline & & & & $(9.8 \pm 0.2) \times 10^{-6}$ & $7.5 \ldots 8.1 \times 10^{-14}$ & $1.0 \ldots 1.1 \times 10^{33}$ & $\sim-6.1$ \\
\hline \multicolumn{2}{|l|}{ Pistol Star } & & & $<1.5 \times 10^{-6}$ & $<5.6 \times 10^{-15}$ & $<7.8 \times 10^{33}$ & $<-5.9$ \\
\hline \multicolumn{2}{|l|}{ WR102ka } & & & $<2 \times 10^{-6}$ & $<7.4 \times 10^{-15}$ & $<5.0 \times 10^{33}$ & $<-6.4$ \\
\hline \multicolumn{2}{|l|}{ GCIRS 16NE } & & & $<3.7 \times 10^{-6}$ & $<1.4 \times 10^{-14}$ & & \\
\hline \multicolumn{2}{|l|}{ GCIRS 16C } & & & $<3.7 \times 10^{-6}$ & $<1.4 \times 10^{-14}$ & $<9.1 \times 10^{33}$ & $<-5.5$ \\
\hline \multicolumn{2}{|l|}{ GCIRS 16SW } & & & $<3.7 \times 10^{-6}$ & $<1.4 \times 10^{-14}$ & & \\
\hline \multicolumn{2}{|l|}{ GCIRS 16NW } & & & $<3.7 \times 10^{-6}$ & $<1.4 \times 10^{-14}$ & $<9.1 \times 10^{33}$ & $<-5.5$ \\
\hline \multicolumn{2}{|l|}{ GCIRS 33SE } & & & $(1.41 \pm 0.02) \times 10^{-5}$ & $(1.42 \pm 0.02) \times 10^{-13}$ & $(1.23 \pm 0.02) \times 10^{33}$ & $-6.244 \pm 0.006$ \\
\hline \multicolumn{2}{|l|}{$\begin{array}{c}\text { 2MASS J17460562 } \\
-2851319\end{array}$} & & & $<1.2 \times 10^{-6}$ & $<4.4 \times 10^{-15}$ & $<6.2 \times 10^{33}$ & $<-6.0 \ldots .6 .4$ \\
\hline
\end{tabular}

Notes. A "H" after the Obs_ID indicates HRC data, the off-axis angle (OFA) is the distance to the non-offset aimpoint. Column 1 provides the (c)LBV name, Cols. 2 to 4 the details of the observation used, and the next columns present the derived count rates, observed fluxes, and absorptioncorrected luminosities in the $0.5-8.0 \mathrm{keV}$ energy range. For the detected objects, the quoted errors correspond to $1-\sigma$; for non-detections, the quoted limits correspond to a $90 \%$ chance of being detected were the source brighter. (a) One additional observation $($ seqnum $=500598$, obsid $=6224$ ) covered the region containing this cLBV, but at a very wide off-axis angle: the presence of a neighboring bright pulsar prevents any detection at this position. ${ }^{(b)}$ Two additional observations (seqnum $=500759$, obsid $=7630$ and 9754 ) covered the region that contains this cLBV, but the source lies on the CCDs outer edges.

understood (Davidson \& Humphreys 1997)! Its fame comes from the two impressive brightenings observed in the 19th century (the "Great Eruption" in mid-19th century and the smaller event of 1890). Since then, there has been a lot of discussion about the variability of this star, and its potential recurrence timescale. A period of $5.5 \mathrm{yr}$ was finally identified by Damineli (1996), and it is now detected from radio to X-ray energies.

Though there is no direct evidence for a companion, this timescale is often interpreted as the orbital period of an eccentric binary (for a review see Corcoran 2011). Indeed, a colliding-wind binary (CWB) can easily explain many properties of the observed variability (e.g. the nebula's changes are related to photo-excitation variations linked to the collision effects), though an exact modeling reproducing all observed details is generally difficult (see e.g. in X-rays the work of Parkin et al. 2011).

In the X-ray domain, it is quite remarkable that eta Car has been one of the first massive stars detected at these energies (Seward et al. 1979), soon after a few Cygnus OB2 stars (see Sect. 4.1 .3 below). It has been intensively studied since then, and the observed hardness of the emission and its variability properties have indicated a strong similarity with the well-known CWB WR 140 (for recent campaigns on these objects, see Corcoran 2011; Williams 2011). An extensive RXTE monitoring (Corcoran 2005; Corcoran et al. 2010) notably showed that the X-ray emission appears fairly stable around apastron, but rises before periastron, as the two stars come closer together. At periastron, an abrupt decrease occurs, and this short minimum state is often interpreted as an occultation or eclipse (combined with a switch-off, Parkin et al. 2011) of the CWB emission. The X-ray lightcurve is not strictly periodic, however. The 2003.5 event was more luminous than the 1998 event, and the recent 2009 event displayed a lower and softer flux before periastron, as well as a shorter minimum state (Corcoran 2005; Corcoran et al. 2010). This may be linked to changes in the wind density, through variations of the wind velocity, mass-loss rate, or both (Corcoran et al. 2010). The observed luminosities (in the $2-10 . \mathrm{keV}$ range) vary from $2 \times 10^{35} \mathrm{erg} \mathrm{s}^{-1}$ at maximum to values 50 to 100 times lower at minimum (Ishibashi et al. 1999; Corcoran et al. 2010). Because the actual values of the absorbing column and bolometric luminosity are still debated, it is difficult to give accurate $\log \left[L_{\mathrm{X}} / L_{\mathrm{BOL}}\right]$, but eta Car may certainly 
reach values of -5 . An example of its X-ray spectrum is shown in Fig. 1.

\subsubsection{W 243}

Using two Chandra observations, Clark et al. (2008) studied the $\mathrm{X}$-ray emission of stars in the Westerlund 1 cluster. These authors mention a "weak detection" for W243: their Table 4 lists $7 \pm 6$ net counts for this object, corresponding to a luminosity of about $10^{32} \mathrm{erg} \mathrm{s}^{-1}$ according to their count-to-flux conversion rate $^{8}$.

We re-analyzed the same Chandra data with a different technique (see Sect. 3.2) and we also appraised the many XMM-Newton datasets encompassing the source.

Because the Chandra data show that there is a source at $7.5^{\prime \prime}$ south of W243 (CXOU J164707.6-455235), we did not use an annulus to estimate the background but a nearby circle that is devoid of sources (see Sect. 3.2). When we extracted the data at the position of W243 in both Chandra datasets and combined them, we found 34 net counts for the source if the source region has $5^{\prime \prime}$ radius, and 15 net counts if we reduced the extraction radius to 3.4 (to avoid contamination by the neighbor). This leads to count rates of $0.00061 \pm 0.00015$ and $0.00029 \pm 0.00010 \mathrm{cts} \mathrm{s}^{-1}$, respectively. These results are compatible (e.g. count rates are within $3 \sigma$ ), but only the first case constitutes a formal detection with our definition (see Sect. 3.1 and Fig. 1). The region used in the first case is also the one most prone to contamination from the neighboring object. Indeed, changing the extraction radius to $3.4^{\prime \prime}$ yields similar results for other Chandra targets. We therefore consider the count rate as uncertain, advocating for a "weak detection" status at best.

All XMM-Newton data were taken with the medium filter except for the first two datasets $(0404340101-1240$ and 0311792001 - 1243), which we therefore excluded from the mosaicking process. There is no trace of the source south of W243. Indeed, this source and W243 both lie on the PSF wing of the pulsar PSR J1647-4552, which is situated at 31" east of W243. The presence of this bright X-ray source severely limits the sensitivity achieved at the position of W243. Therefore, because the LBV seems undetected in the XMM-Newton data, an upper limit on its flux was derived by adding a fake source, with a similar PSF, at the position of the $\mathrm{LBV}^{9}$. We found that an X-ray source $\sim 400$ times fainter than the pulsar's emission would be barely discernible, implying an upper limit on the EPIC count rate of $0.0017 \mathrm{cts} \mathrm{s}^{-1}$ (Table 2). Note that because the pulsar was brighter in the two excluded observations, we obtained a better sensitivity limit here than when considering the whole $X M M-N e w t o n$ set. The limit derived from XMM-Newton data is quite similar to the values found using the weak Chandra detection, which means that XMM-Newton is on the verge of detecting W243. However, the Chandra observatory appears ro be better suited for the purpose of studying W243 - deeper observations would help resolve the uncertainties on its properties.

In any case, the modest X-ray emission level of $10^{32} \mathrm{erg} \mathrm{s}^{-1}$ observed in W243 is quite typical of OB stars (Harnden et al. 1979; Ku \& Chanan 1979; see also recent cluster studies of Sana et al. 2006; Antokhin et al. 2008; Nazé et al. 2011a), as well as

\footnotetext{
8 See their Table 4; this probably corresponds to a conversion toward absorption-corrected flux, though it is not clearly indicated in the paper. 9 The astrometric errors of the XMM-Newton datasets are negligible because the error on the pulsar's position, found by comparing the Simbad coordinates with those in the detection list of the combined mosaic, amounts to only $0.2^{\prime \prime}$.
}

its $\log \left[L_{\mathrm{X}} / L_{\mathrm{BOL}}\right]$ of -7 (Pallavicini et al. 1981; Berghoefer et al. 1997; Nazé 2009, 2011). This leads to two possibilities. On the one hand, W243 could be a single LBV with a wind comparable to that of a "normal" O-type star. While its mass-loss rate is indeed similar to that of O-type stars, the wind terminal velocity of W243 was found to be only $165 \mathrm{~km} \mathrm{~s}^{-1}$, i.e. one order of magnitude lower than in O-stars (Ritchie et al. 2009). Such a slow wind casts doubt on the "LBV wind-shock model" scenario, because the (potential) intrinsic shocks will be fairly weak to generate enough X-rays, compared to O-stars. On the other hand, W243 could be a multiple system composed of an LBV and an O-type star whose intrinsic high-energy emission is the actual source of the recorded X-rays, and not the LBV itself.

\subsubsection{NAME VI Cyg 12 = Cyg OB2 \#12}

With $M_{V} \simeq-10$, Cyg OB2 \#12 is considered to be one of the optically brightest stars in our Galaxy (e.g. Massey \& Thompson 1991). Despite this high luminosity, which places it above the Humphreys-Davidson limit, Cyg OB2 \#12 lacks some of the LBV characteristics, such as temperature changes, enrichment, or traces of circumstellar material (Clark et al. 2011). This object was one of the very first massive stars detected as an X-ray source during an EINSTEIN observation of Cyg X-3 (Harnden et al. 1979). This star has subsequently been observed by all major X-ray satellites. Most recently, Rauw (2011) performed an in-depth monitoring of this object using six XMM-Newton pointings obtained in October-November 2004 and April-May 2007. The EPIC spectra of this star (Fig. 1) appeared to be quite hard: it was fitted with an absorbed two-temperature thermal plasma model consisting of plasma at temperatures of $k T \simeq 0.75$ and $2 \mathrm{keV}$ (where the strength of the second component is quite similar to that of the first one, not much weaker). The average absorption-corrected X-ray flux in the $0.5-8.0 \mathrm{keV}$ domain was $2.4 \times 10^{-11} \mathrm{erg} \mathrm{cm}^{-2} \mathrm{~s}^{-1}$, which corresponds to an X-ray luminosity of $8.2 \times 10^{33} \mathrm{erg} \mathrm{s}^{-1}$ assuming a distance of $1.7 \mathrm{kpc}$ and $\log \left[L_{\mathrm{X}} / L_{\mathrm{BOL}}\right]=-6.1$ (using reddening, distance and bolometric luminosity of Table 1 rather than the values used by Rauw 2011 - though the final $\log \left[L_{\mathrm{X}} / L_{\mathrm{BOL}}\right]$ value are then equal).

These temperatures, especially the second one, cannot be explained by shocks in a slow $\left(150 \mathrm{~km} \mathrm{~s}^{-1}\right)$ moving wind as proposed by Klochkova \& Chentsov (2004) and more recently by Clark et al. (2011, $400 \mathrm{~km} \mathrm{~s}^{-1}$ ). However, Souza \& Lutz (1980) reported a variable absorption blueward of $\mathrm{H} \alpha$ that they tentatively interpreted as a blueshifted $\mathrm{H} \alpha$ absorption arising in an expanding shell at a velocity of $1400 \mathrm{~km} \mathrm{~s}^{-1}$. Leitherer et al. (1982) inferred this velocity to correspond to the wind velocity of Cyg OB2 \#12. An expanding shell of even higher velocity (around $3100 \mathrm{~km} \mathrm{~s}^{-1}$ ) was reported by Wang \& Zhu (2003). A faster wind than inferred by Klochkova \& Chentsov (2004) is therefore possible, though the tremendous interstellar reddening unfortunately hampers a reliable determination of the star's wind velocity.

On the other hand, the EPIC data furthermore revealed X-ray flux variability (with an amplitude of $\sim 10 \%$ ) on timescales ranging from a few days to a few weeks, as well as stronger variations $(37 \%)$ on timescales of years (Rauw 2011). The combination of these properties (strong, variable X-ray emission, including a strong high-temperature component) suggest that Cyg OB2 \#12 could be either an eccentric colliding wind binary (with a secondary star featuring a fast wind) or a single star featuring a strong magnetic field that confines the stellar wind near the magnetic equator. 

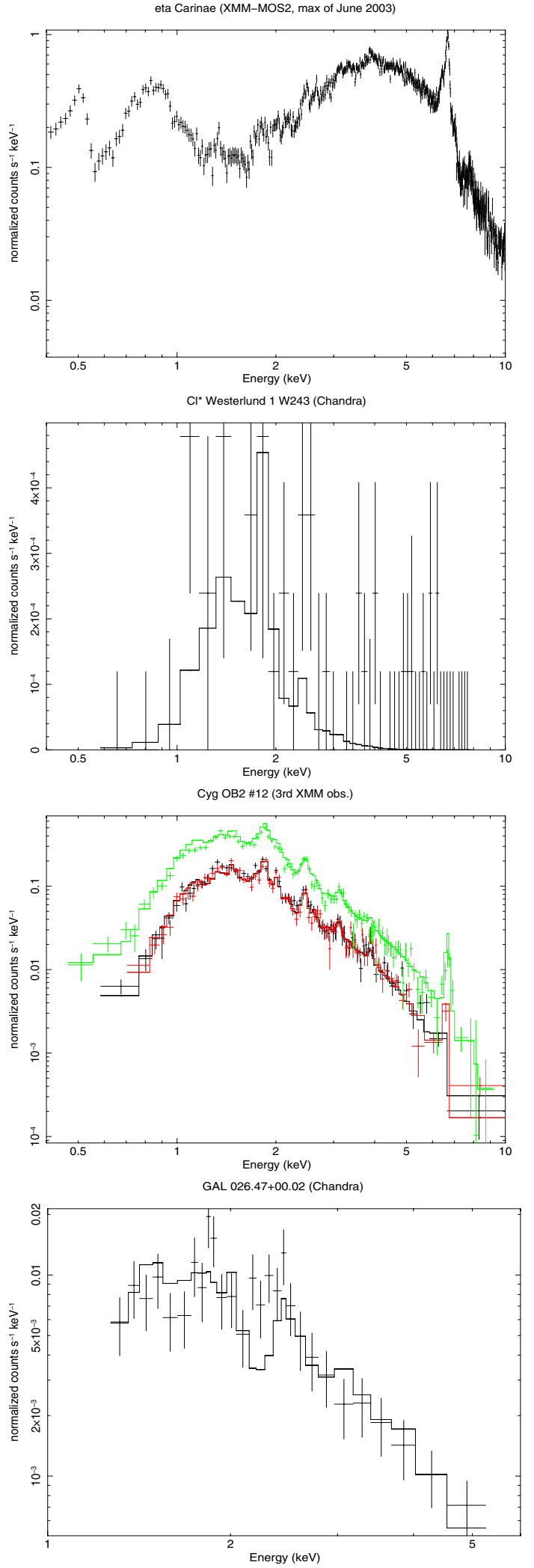

Fig. 1. X-ray spectra of the detected LBVs (from top to bottom: eta Carfrom the 2XMM database, W243, Cyg OB2 \#12 - from Rauw 2011, and GAL 026.47+00.02), along with their best-fit model (see text for details). The eta Car spectrum corresponds to XMM-MOS2 data taken at the June 2003 maximum, which are dominated by the soft emission of the outer ejecta below $1.5 \mathrm{keV}$ and by colliding-wind emisson above that limit (Hamaguchi et al. 2007). The W243 spectrum corresponds to the $5^{\prime \prime}$ extraction region, and was re-binned by a factor of 10 . Note the strong iron line at $6.7 \mathrm{keV}$ for eta Car and Cyg OB2 \#12.
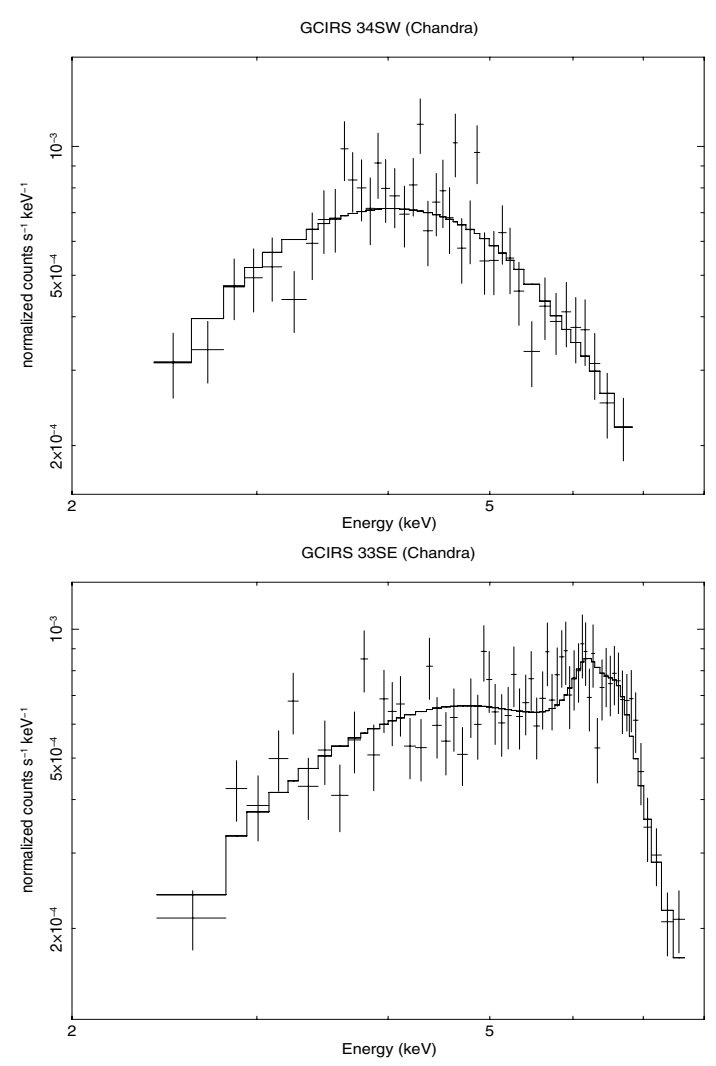

Fig. 2. X-ray spectra of the X-ray sources apparently associated with GCIRS 34W and GCIRS 33SE, along with their best-fit model (see text for details). Note the absence of lines, especially compared to Fig. 1.

\subsubsection{GAL $026.47+00.02$}

GAL 026.47+00.02 was detected by Chandra as a rather faint $\mathrm{X}$-ray source (only $\sim 300$ counts recorded in $20 \mathrm{ks}$ ). This X-ray source is isolated, which makes its association with the cLBV credible.

We were able to extract a rough (in view of the $\mathrm{S} / \mathrm{N}$ ) X-ray spectrum for this object (Fig. 1). A fit by an absorbed optically thin thermal plasma model yields a temperature of $1.47 \pm$ $0.11 \mathrm{keV}$ and a normalization factor of $(5.7 \pm 0.4) \times 10^{-4} \mathrm{~cm}^{-5}$ when the absorption is fixed to the ISM value. The resulting $\log \left[L_{\mathrm{X}} / L_{\mathrm{BOL}}\right]=-6$ is similar to that of Cyg OB2 \#12, though the former object appears somewhat softer. This bright X-ray emission suggests that there are colliding winds or magnetically confined winds in the system. However, follow-up observations (e.g. to test whether the X-ray source varies, and how) would be required to constrain the nature of GAL $026.47+00.02$.

\subsection{Doubtful detections}

\subsubsection{GCIRS 34W}

This LBV appears at $0.3^{\prime \prime}$ of the X-ray source CXOUGC J174539.7-290026 in the Galactic center survey (Muno et al. 2009). However, the X-ray source does not appear point-like but rather looks like a diffuse emission region. This casts doubts on the identification of the source of the recorded X-rays with the sole LBV.

This conclusion is supported by the appearance of the X-ray spectrum (Fig. 2). An absorbed optically-thin thermal plasma provides quite a good fit, but only with extremely high temperatures: $k T>53 \mathrm{keV}$, norm $=(1.09 \pm 0.05) \times 10^{-4} \mathrm{~cm}^{-5}$, and 
an absorption fixed to the ISM value yield $\chi^{2}=1.5$. An absorbed powerlaw is clearly better: $\Gamma=0.95 \pm 0.16$, norm $=$ $(1.1 \pm 0.2) \times 10^{-5} \mathrm{~cm}^{-5}$, and an absorption fixed to the ISM value yield $\chi^{2}=1.1$. Both models yield quite similar flux values, though (both are given in Table 3 ).

\subsubsection{GCIRS 33SE}

The cLBV is at $0.3^{\prime \prime}$ of the X-ray source CXOUGC J174540.0290030 of the Galactic center survey (Muno et al. 2009). This source was found to vary in flux by two orders of magnitude (from photon fluxes of $1.6 \times 10^{-6} \mathrm{ph} \mathrm{cm}^{-2} \mathrm{~s}^{-1}$ to $2.3 \times$ $10^{-4} \mathrm{ph} \mathrm{cm}^{-2} \mathrm{~s}^{-1}$ ).

The mean spectrum appears to be extremely hard, with all photons at energies higher than $2 \mathrm{keV}$ (Fig. 2). We were unable to fit it with an absorbed powerlaw, an absorbed optically-thin thermal plasma, an absorbed bremsstrahlung, or an absorbed disk blackbody model. Indeed, a good fit was only achieved by adding a flat powerlaw to a line (either a Gaussian or a relativistic one): for the latter case (model diskline in Xspec), we found $\Gamma=-0.08 \pm 0.12$, norm $_{\mathrm{PL}}=(2.2 \pm 0.6) \times 10^{-6} \mathrm{~cm}^{-5}, E_{\text {line }}=$ $6.41 \pm 0.05 \mathrm{keV}, i>77^{\circ}$, and norm $_{\text {line }}=(4.8 \pm 0.4) \times 10^{-6} \mathrm{~cm}^{-5}$.

This type of spectrum and variations are quite atypical of massive stars (even considering the "hard" colliding-wind systems) and would better fit in the context of X-ray binaries. Therefore, we doubt that the survey has detected the true emission of GCIRS 33SE.

\subsection{Non-detections}

\subsubsection{P Cyg}

The RosAT data of P Cyg were analyzed by Berghöfer \& Wendker (2000) and Oskinova (2005a). However, these X-ray data suffer from strong contamination by the intense UV emission from this hot star, which implies most probably a non-detection of PCyg in this dataset. Limiting (absorption-corrected) luminosities of $9 \times 10^{30} \mathrm{erg} \mathrm{s}^{-1}$ and $2 \times 10^{31} \mathrm{erg} \mathrm{s}^{-1}$ in the RosaT range $(0.1-2.5 \mathrm{keV})$ were reported by Berghöfer \& Wendker (2000) and Oskinova (2005a), respectively. This corresponds to $\log \left[L_{\mathrm{X}} / L_{\mathrm{BOL}}\right]$ below $\sim-8$ (considering the same soft energy range).

The new XMM-Newton observation was taken with the thick filter to avoid any UV contamination. In the new data, three faint $\mathrm{X}$-ray sources are clearly detected toward the east of P Cyg. They are at distances of $28^{\prime \prime}$ (for two of them) and 62" (for the last one of the trio). This is well above the potential astrometric errors and the PSF size, i.e. these source are too distant to be reasonably associated with the LBV.

Definitely, there is no X-ray source at the position of P Cyg. The upper limit on the flux is more than an order of magnitude lower than the ROSAT limit, though it corresponds to a harder energy range (0.5-8. keV for XMM-Newton vs $0.1-2.5 \mathrm{keV}$ for ROSAT). The signal recorded by RosAT was therefore clearly dominated by UV contamination.

\subsubsection{AG Car}

The field of AGCar looks very similar to that of PCyg: only faint X-ray sources scattered everywhere, without anything at the position of the LBV. Here, the closest X-ray source lies at nearly $1^{\prime}$ of AGCar, and there is consequently no doubt on the non-detection of the LBV.
Note that Hen 3-519 is fairly close to AG Car, but no constraint on its X-ray flux can be derived from these data, because it is out of the field-of-view of the MOS cameras and just on the edge of a pn CCD.

\subsubsection{HD 160529}

The XMM-Newton observation appears more densely populated than in the cases of PCyg and AGCar, but the closest X-ray source appears at 56" of HD 160529 and, again, the LBV clearly remains undetected.

\subsection{4. $\mathrm{qF} 362$}

The deep Galactic center survey data do not show any X-ray source in the vicinity of $\mathrm{qF} 362$. The closest X-ray source, CXOUGC J174616.6-284909, lies at more than 18", which is clearly too far away from the LBV considering the narrow PSF of Chandra.

\subsubsection{GAL 024.73+00.69}

A strong flare affected the XMM-Newton observation, reducing the useful exposure to only $3.5 \mathrm{ks}$. This indeed limits the sensitivity of the observations: only $7 \mathrm{X}$-ray sources were detected in the field-of-view and none appears close of the LBV (they are at separations $>7^{\prime}$ ).

\subsubsection{Pistol star}

Before the publication of the full Galactic center survey, Muno et al. (2006) and Oskinova (2005b) analyzed the then available Chandra exposures of the region, and they report a limiting $\log \left[L_{\mathrm{X}} / L_{\mathrm{BOL}}\right]$ of -8.2 and -8 , respectively.

The deep Galactic center survey data do not show any X-ray source in the vicinity of Pistol star. The closest X-ray source, CXOUGC J174615.2-284957, lies at about 6". The derived limit on the $\log \left[L_{\mathrm{X}} / L_{\mathrm{BOL}}\right]$ is -6 , which may seem at odds with previous reported results, though the survey of Muno et al. (2009) uses more datasets. Though no detail on the exact derivation of the $\log \left[L_{\mathrm{X}} / L_{\mathrm{BOL}}\right]$ limit is provided in these previous papers, it is interesting to note that these limits are found if one uses the observed flux, not the absorption-corrected one: because the absorption in the direction of the Galactic center is high, this explains the apparent discrepancy of two orders of magnitude in $\log \left[L_{\mathrm{X}} / L_{\mathrm{BOL}}\right]$. However, to compare objects scattered all over the Galaxy, it is important to correct the luminosities for the interstellar absorption - indeed, the "canonical" $\log \left[L_{\mathrm{X}} / L_{\mathrm{BOL}}\right]=-7$ of O-stars only applies if this correction is made. Unfortunately, this results in a much weaker constraint on the X-ray emission of the Pistol star.

\subsubsection{HD 316285}

Data in the vicinity of this cLBV were collected by $X M M-N e w t o n$ during three observations. In the observation $0112970201-0145$, the LBV appears to be so far off-axis that it is out of the field-of-view of MOS2. In addition, there are a lot of flares in $0205240101-0956$. This probably explains why the limiting count rate derived with only the $0112970101-0145$ dataset is more constraining than the limit derived when considering all three datasets. We therefore quote in Table 2 the former value only. 


\subsubsection{GRS $25.5+0.2$}

This cLBV was observed quite far off-axis $\left(10^{\prime}\right)$ in a relatively short $(7.5 \mathrm{ks})$ exposure: the sensitivity is therefore quite shallow, and the derived limit on the count rate is accordingly high. The closest X-ray source appears at $4^{\prime}$ of the cLBV, excluding any confusion problems.

\subsubsection{WR102ka}

The deep Galactic center survey data do not show any X-ray source in the vicinity of WR102ka. The closest X-ray sources, CXOUGC J174616.6-290115 and J174618.3-290204, lie at about 28 ".

\subsubsection{LBV $1806-20$}

This cLBV is situated 14" east of the bright pulsar SGR 180620. While this separation is no problem for Chandra, the cLBV is situated on the PSF wing of this bright pulsar in XMM-Newton instruments. We made a mosaic with all available XMM-Newton observations, except the last one $(0604090201-1785)$, which was taken with a different filter, but the produced sensitivity map has no actual meaning. The situation is even worse than for W243 because the separation is smaller: the detection is mostly hampered by the closeness of the pulsar rather than by the available effective area. Therefore, since the cLBV seems undetected, we estimated the true detection limit by adding a fake source with a similar PSF at the position of the $\mathrm{CLBV}^{10}$. We changed its amplitude until it was not detectable. We found that an X-ray source 100 times fainter than the pulsar's emission would be barely discernible: this constitutes our flux limit for XMM-Newton, and corresponds to an EPIC count rate of $0.01 \mathrm{cts} \mathrm{s}^{-1}$ (Table 2).

For Chandra, there is no confusion problem, but the pulsar left a readout trail in the ACIS data. The background was therefore not measured in an annulus, but instead taken in a circle of the same size as the source region and that lies on the same readout trail. This resulted in the limit quoted in Table 3. The same regions were indeed used for the HRC data, for coherence reasons. Note that the cLBV is formally detected at $1 \sigma$ significance (i.e. not an actual detection with our criteria, see Sect. 3.1) in the ACIS data, but this is clearly an artifact caused by the readout trail. The Chandra observations are better suited to separate the pulsar from the cLBV, but unfortunately, they are not very sensitive, and do not improve the XMM-Newton results.

\subsubsection{Sher 25}

Using a single observation of NGC 3603, Moffat et al. (2002) reports a non-detection of Sher 25, without any detail on the limiting X-ray flux.

The additional recent observations enable us to enhance the sensitivity in the region by an order of magnitude, which results in a tight upper limit on the X-ray emission of Sher25. Note that the source is formally detected with $1 \sigma$ significance (i.e. not an actual detection with our criteria, see Sect. 3.1) because there are $3.3 \pm 3$ net counts in the source region.

\footnotetext{
${ }^{10}$ Note that the astrometric errors of the XMM-Newton datasets are very limited because the error on the pulsar's position, found by comparing the Simbad coordinates with those in the detection list of the combined mosaic, amounts to only $0.2^{\prime \prime}$.
}

\subsubsection{2. [OMN2000] LS1}

Situated in the outskirts of the massive star-forming region W51A, LS1 shows a formal $1 \sigma$ "detection" (i.e. not an actual detection with our criteria, see Sect. 3.1), with an upper limit on the observed flux of $1.4 \times 10^{-15} \mathrm{erg} \mathrm{cm}^{-2} \mathrm{~s}^{-1}$.

\subsubsection{GCIRS 16NE, 16C, 16SW, 16NW}

The deep Galactic center survey data do not show any X-ray source at the position of these objects. The closest X-ray sources are GCIRS 33SE and 34SW (see above), as well as CXOUGC J174540.1-290025, J174540.0-290028 and J174539.7-290029. We used the same absorbing column for all sources to derive the limiting flux.

This region of the survey is the one observed with the highest sensitivity, which explains why the limiting photon fluxes are so low. However, this is also the most crowded region of the survey: the derived limits may therefore be somewhat optimistic, although it is difficult to quantify by how much.

\subsubsection{MASS J17460562-2851319}

The deep Galactic center survey data do not show any X-ray source in the vicinity of this target. The closest X-ray source, CXOUGC J174604.4-285139, lies at about 17".

\subsubsection{MCW 930}

The position of this cLBV falls in a gap of the pn camera. Only MOS data were therefore used to derive an upper limit on its $\mathrm{X}$-ray emission. The closest X-ray source appears more than $3^{\prime}$ away from the cLBV. There is therefore no doubt on the nondetection of this object.

\subsubsection{MASS J17110094-3945174}

Situated in a field containing only a few faint X-ray sources, this object remains undetected in the XMM-Newton observation. While a flare affected the exposure, especially the pn data, the target was observed nearly on-axis, leading to a fairly deep upper limit. Unfortunately, as for the other objects of Wachter et al. (2010) and Gvaramadze et al. (2010b), no information on the other properties of the object are available, preventing the derivation of a $\log \left[L_{\mathrm{X}} / L_{\mathrm{BOL}}\right]$.

\subsubsection{MASS J18415965-0515409}

No bright X-ray source is present in the field-of-view of the Chandra observation, and no X-ray source lies closer than 1' away from 2MASS J18415965-0515409. A flux limit of $1.3 \times$ $10^{-15} \mathrm{erg} \mathrm{cm}^{-2} \mathrm{~s}^{-1}$ was derived.

\subsubsection{MASS J16461734-4508478}

This source is undetected in the XMM-Newton observation. The sole X-ray source of the field-of-view appears at 3.6'. Because the target was observed not far from the on-axis position (off-axis angle $=2.6^{\prime}$ ), we were able to put a good constraint on its count rate. 


\subsubsection{MASS J18022233-2238002}

The region encompassing 2MASS J18022233-2238002 was observed three times by XMM-Newton. However, two of these datasets (0135743001 - 0600 and $0135743101-0607)$ have a bug in their attitude files, which could not be corrected (even with the help of the XMM-Newton helpdesk). Therefore, no mosaicking was possible for this target. We instead used a reduction process similar to the one applied for Chandra data: we first derived the number of counts appearing in the source and background regions for each dataset by extracting data in adequate zones (two neighboring circles of $30^{\prime \prime}$ radii), then we found the actual exposure times for each dataset at the position of the target using individual exposure maps, and finally we used the CIAO task aprates to derive the $90 \%$ detection limit on the count rate. However, the two additional datasets did not improve the upper limit found when using only the $0135742801-0600$ exposure, probably because of the flares affecting the other Rev. 0600 data and because the target appears in a gap of the pn camera for Rev. 0607. Table 2 therefore quotes the value derived using only the first dataset.

\subsubsection{MN53}

This object was observed by both XMM-Newton and Chandra. Both observations were centered on the bright 1RXS J170849.04009 , and the field contains a few additional faint sources (detected only with XMM-Newton). MN53 remains undetected, the closest X-ray source appearing at 70" in the XMM-Newton data. Note that the pn data are limited to a small area around the main target, so that the flux limit was derived using MOS data only. In the Chandra data, MN53 is formally detected at $1 \sigma$ significance (i.e. not an actual detection with our criteria, see Sect. 3.1) and the flux limit derived on this HRC observation is about 20 times higher than that found from the XMM-Newton data of the same region.

\subsubsection{MN79}

MN79 appears in a sparsely populated region that contains only faint X-ray sources. The closest one in the XMM-Newton data lies at $47^{\prime \prime}$ of the target and at $15^{\prime \prime}$ in the Chandra data, but the target itself remains undetected. The two observatories provided similar flux limits.

\subsubsection{MN83}

Situated at the other side of the aimpoint compared to GAL $026.47+00.02$, MN83 shows a contrasting behavior by remaining totally undetected. The closest X-ray source is at $\sim 30^{\prime \prime}$, and the limiting count rate derived for MN83 is about two orders of magnitude below that of GAL 026.47+00.02.

\section{Discussion}

This first LBV survey in the X-ray domain covers half of the known LBVs or candidates (or two-thirds of the catalog of Clark et al. 2005b). Despite the heterogeneity of the dataset, the derived limits in observed fluxes are remarkably similar, lying between $10^{-15}$ and $10^{-14} \mathrm{erg} \mathrm{cm}^{-2} \mathrm{~s}^{-1}$. However, because the distance and reddening of the surveyed objects vary a lot, so do the constraints on the X-ray luminosity of LBVs: the derived limits span more than four orders of magnitude, from $\sim 10^{30}$ to $\sim 7 \times 10^{34} \mathrm{erg} \mathrm{s}^{-1}$.
Of all surveyed objects, only four are detected in X-rays, corresponding to a detection fraction of one out of four in the sample of confirmed LBVs, one out of nine for the Clark's catalog, or one out of 17 for the full sample of (c)LBVs. In addition to the two well-known cases of eta Car and Cyg OB2\#12, GAL $026.47+00.02$ and W243 were found to emit some X-rays. Two additional cases are formally detected, but the properties of their X-ray emission cast doubt on the identification of the high-energy source with the (c)LBVs.

With the results of our survey at hand, what can be concluded about the X-ray emission of LBVs?

First, LBVs, as a class, are clearly not bright X-ray emitters. Moreover, it seems unlikely that LBVs possess an intrinsic $\mathrm{X}$-ray emission of a level comparable to that of O-type stars, which are moderate X-ray emitters. While O-stars display a tight relation between their bolometric and X-ray luminosities $\left(\log \left[L_{\mathrm{X}} / L_{\mathrm{BOL}}\right] \sim-7\right.$ with dispersions down to 0.2 dex for clusters, see cluster studies of Sana et al. 2006; Nazé et al. 2011a; and discussions in Nazé 2009, 2011), five (c)LBVs in our sample have $\log \left[L_{\mathrm{X}} / L_{\mathrm{BOL}}\right]<-8.2$, P Cyg even showing no emission at our sensitivity limit of $\log \left[L_{\mathrm{X}} / L_{\mathrm{BOL}}\right]-9.4$ ! This indicates a significantly weaker X-ray emission, compared to O-stars, for the LBVs as a class. A quite similar conclusion was found for WolfRayet stars, especially those of the WC type (Oskinova et al. 2003). This could have two reasons: either the local absorption by the wind is too high to let any X-rays generated in the deep inner layers escape, like for WC stars, or the wind velocity is too low (a few hundred $\mathrm{km} \mathrm{s}^{-1}$, as is often observed in LBVs) to produce hot plasma through intrinsic wind shocks. While both may well explain the observed lack of X-rays in LBVs, it is unfortunately difficult to accurately constrain the wind velocity (see e.g. the case of Cyg OB2 \#12 discussed above) or absorption (see e.g. the case of eta Car, Corcoran 2011), whatever the phase of LBV activity. Both hypotheses (and a combination of the two) therefore remain viable at the present time.

If LBVs are intrinsically X-ray faint, the few cases of X-ray detections should have an extrinsic cause. The most obvious candidate is binarity. It is indeed well known that wind-wind collisions in massive binaries (i.e. $\mathrm{O}+\mathrm{OB}$ or $\mathrm{WR}+\mathrm{OB}$ ) can produce bright, hard and variable X-ray emission (for a review, see Güdel \& Nazé 2009). This is certainly the case for the Galactic LBV eta Car (see above) and the SMC LBV HD 5980 (Nazé et al. 2007, and references therein): the phase-locked variations of their bright and hard X-ray emission are a definite proof of colliding winds. Such a process has also been strongly advocated for Cyg OB2 \#12 in view of its spectral properties and its luminosity changes (see Sect. 4.1.3 above), though a direct evidence for periodicity in the variations is still lacking. In addition, the bright X-rays of GAL $026.47+00.02$ are again reminiscent of a wind-wind collision but additional X-ray data are needed to unveil the variability properties of the X-ray emission and ascertain the nature of this cLBV (Sect. 4.1.4). Finally, W243 does not show any sign of colliding wind X-rays, but its $\mathrm{X}$-ray emission may well be that of an O-type companion (see Sect. 4.1.2 above). For the four detected (c)LBVs, binarity constitutes a plausible explanation, even if other processes, such as magnetically-confined winds, cannot be formally ruled out.

Does that mean that other (c)LBVs are single, because they are not X-ray bright? It must be recalled that both Chandra and XMM-Newton have shown that X-ray bright CWBs are the exception, not the rule (Sana et al. 2006; Nazé 2009; Nazé et al. 2011a). In addition, even in the rare cases of X-ray bright CWBs, the X-ray emission may not be as bright and hard throughout the whole orbit (for a review, see Güdel \& Nazé 2009). Therefore, 
while a bright, hard and phase-locked X-ray emission may be a strong indication of binarity, the opposite is certainly not true. The presence of a companion cannot always be inferred from $\mathrm{X}$-ray studies, and the single status of the other (c)LBVs of our study cannot be definitively established on the sole basis of their apparent lack of bright X-rays at the time of the observation(s).

Our study provides additional information that allows one to constrain the presence of an early OB companion because these stars are not X-ray dark. They display typical X-ray luminosities of $10^{31}$ to $10^{33} \mathrm{erg} \mathrm{s}^{-1}$ (Harnden et al. 1979; Ku \& Chanan 1979; see also recent statistical studies of Sana et al. 2006; Antokhin et al. 2008; Nazé 2009; Nazé et al. 2011a). Even if bright $\left(>10^{33} \mathrm{erg} \mathrm{s}^{-1}\right)$ wind-wind collisions do not exist in massive systems, the intrinsic X-ray emission of the companion may show up, because the luminosity ratio is less favorable for the LBV at these high energies. Unfortunately, many of our luminosity limits are quite high, hence not always able to test this scenario. Only in five cases (P Cyg, AG Car, HD 160529, HD 316825, and Sher 25) are the data sufficiently sensitive to probe the range of $\mathrm{X}$-ray luminosities of $\mathrm{OB}$ stars, taking into account the interstellar absorption of our targets.

If these LBVs possess OB companions, there are basically two possibilities: either (1) the companion is quite close to the LBV or (2) the companion is relatively distant from the LBV. In the first case, the intrinsic emission of the companion may be hidden by the strong absorption of the dense wind, but then the conditions are favorable to bring about an X-ray bright wind-wind collision. In the second case, no emission from a wind-wind collision is expected owing to wind dilution, but the intrinsic emission of the companion would be easily detectable, because the tenuous (at these distances) wind of the LBV cannot hide it anymore.

To evaluate the limit between these two scenarios, we focused on the most constrained case, that of P Cyg. The wind density $\rho(r)$ can be written as $\dot{M} / 4 \pi r^{2} v(r)$ where the velocity is assumed to follow a beta-law with $\beta=1$, i.e. $v(r)=v_{\infty}\left(1-\left(R_{*} / r\right)\right)$. In this case, the equivalent absorbing column for hydrogen, from a position $R_{\text {start }}$ in the wind can be written as

$N_{w}(r)=\frac{X_{\mathrm{H}}}{m_{\mathrm{H}}} \int_{R_{\text {start }}}^{\infty} \rho(r) \mathrm{d} r=\frac{X_{\mathrm{H}} \dot{M}}{4 \pi R_{*} v_{\infty} m_{\mathrm{H}}}\left[-\ln \left(1-\frac{R_{*}}{R_{\text {start }}}\right)\right]$.

Using atmosphere models, Najarro et al. (1997) and Najarro (2001) have calculated the stellar parameters of PCyg: $R_{*}=$ $76 R_{\odot}, \dot{M} \sim 2 \times 10^{-5} M_{\odot} \mathrm{yr}^{-1}, n_{\mathrm{He}} / n_{\mathrm{H}}=0.3^{11}$ and $v_{\infty}=$ $185 \mathrm{~km} \mathrm{~s}^{-1}$. With these parameters and $R_{\text {start }}$ of $2,5,10,50$, and $100 R_{*}$, we find absorbing columns of $20,6,3,0.6$, and $0.3 \times$ $10^{22} \mathrm{~cm}^{-2}$, respectively. An optically-thin plasma with a temperature of $0.6 \mathrm{keV}$ and the strength of a late O-early B star (i.e. $10^{31} \mathrm{erg} \mathrm{s}^{-1}$ at a distance of $1.7 \mathrm{kpc}$ ), which would suffer both the interstellar absorption of P Cyg and an absorption by these columns (modeled within Xspec using the vphabs model and a three times solar abundance for helium) would have been detected as long as $R_{\text {start }}>25 R_{*}$. This means that only very close binaries would escape detection, and only in the worst cases of orientation (i.e. O-star seen through the LBV wind, not through the O-star wind). We therefore consider it highly probable that $\mathrm{PCyg}$ is not an LBV+OB binary.

In summary, we have information on the distance, reddening, and X-ray luminosity of 24 objects in our survey (i.e. all studied cases except the Wachter et al. and Gvaramadze et al. candidates). Of these, four appear to be probable binaries and

\footnotetext{
11 Which corresponds to an abundance fraction (in weight) $X_{\mathrm{H}}$ of $\sim 0.45$.
}

five are probably single stars. The status of the remaining objects is unclear: we therefore have between 4 and 19 binaries and between 5 and 20 single stars. Assuming a binary fraction of 0.5 , as is often suggested for massive stars (Sana \& Evans 2011), we modeled the LBV population result by a random picking of stars using a binomial distribution, so that we derived the probability to obtain the different plausible scenarios (i.e. 4 binaries +20 single, 5 binaries +19 single, ... 19 binaries +5 single) . The probability to observe our situation is found to be $99.91 \%$, for the assumed binary fraction of 0.5 . Only binary fractions below 0.26 or higher than 0.69 would lead to a different situation in $10 \%$ of the cases. However, these very low or very high binary fractions seem unlikely for populations of massive objects (Sana \& Evans 2011). Considering only the confirmed LBVs yields similar results, though the sample is smaller and the statistics therefore less good. We did not find any evidence regarding the binary fraction that LBVs are different from other populations of massive objects.

It must be noted, however, that binarity was proposed to play a role in the occurrence of giant eruptions. More specifically, these events were to be triggered by close interactions between the components during some periastron passages. An example would be the 19th-century eruptions of eta Car, which occurred within weeks of periastron (Kashi \& Soker 2010; Smith et al. 2011), and the 20th-century eruptions of HD 5980 (Koenigsberger 2004; Koenigsberger et al. 2010). The last case of historical giant eruptions is P Cyg, and the impact of a companion has also been strongly advocated for this star by Kashi (2010). While our observations most probably rule out the presence of a massive companion, we cannot exclude that a lowermass object lurks in the neighborhood of PCyg. For Kashi (2010), a late-B companion with 3-6 $M_{\odot}$ is sufficient to trigger massive ejections. Such an object would produce no X-ray bright colliding wind emission (since its wind is negligible) nor a large amount of intrinsic X-rays: it would therefore remain undetected even in our sensitive observations! The question therefore remains open as to whether binarity should be considered as a universal trigger of LBV eruptions (see also the discussion of alternative triggers in Smith et al. 2011).

\section{Conclusion}

We have performed the first survey of Galactic LBVs and candidate LBVs in the X-ray domain using a dedicated XMM-Newton dataset as well as archival Chandra and XMM-Newton data. About half of the known (c)LBVs (31 out of 67) or two-thirds of the (c)LBVs listed by Clark et al. (2005b, 22 out of 35) have now been studied in the X-ray domain.

The X-ray emission of six objects is detected, but the association with the (c)LBVs is doubtful for two of them (GCIRS $33 \mathrm{SE}$ and $34 \mathrm{~W})$. We are therefore left with four (c)LBVs with detections in the X-ray range: the well-known eta Car and Cyg OB2 \#12, plus GAL 026.47+00.02 and W243. Because it is bright, GAL 026.47+00.02 appears to be similar to the two "historical" detections (eta Car and Cyg OB2 \#12). These three cases are satisfactorily explained, at least quantitatively, by wind-wind collisions in binary systems. In contrast, the X-ray emission of W243 appears more modest and it is compatible with the typical emission level of OB stars: since the wind velocity of W243 is low, the observed emission may be that of an otherwise hidden OB companion.

Excluding these objects, only upper limits were found, with values down to $L_{\mathrm{X}}=8 \times 10^{29} \mathrm{erg} \mathrm{s}^{-1}$ and $\log \left[L_{\mathrm{X}} / L_{\mathrm{BOL}}\right]-9.4$ for PCyg. The constraints on the X-ray emission are indeed 
particularly strong in five cases (PCyg, AGCar, HD 160529, HD 316825, and Sher 25) where even the usual X-ray emission of a potential OB companion can apparently be excluded, indicating that these stars are probably single and that the intrinsic level of X-ray emission is very low in these LBVs.

Our results (four detections and five strong limits) are fully compatible with binary fractions between 0.26 and 0.69 in LBVs, which agrees well with what is found for $\mathrm{O}$ and WR stars. To the limits of our dataset, the multiplicity properties of LBVs do not present significant deviations from other populations of massive stars. Binarity may have an impact on the occurrence of giant eruptions, however, but additional data are needed in order to fully test this scenario.

Acknowledgements. The authors thank L. Townsley, P. Broos, and L. Bassani very much for sharing their proprietary data. They acknowledge support from the Fonds National de la Recherche Scientifique (Belgium), the Communauté Française de Belgique, the PRODEX XMM-Integral and Herschel contracts, and the "Action de Recherche Concertée" (CFWB-Académie Wallonie Europe). Y.N. also thanks the XMM-Newton helpdesk for interesting discussions on mosaicking data. ADS and CDS were used for preparing this document.

\section{References}

Antokhin, I. I., Rauw, G., Vreux, J.-M., van der Hucht, K. A., \& Brown, J. C. 2008, A\&A, 477, 593

Barniske, A., Oskinova, L. M., \& Hamann, W.-R. 2008, A\&A, 486, 971

Berghöfer, T. W., \& Wendker, H. J. 2000, Astron. Nachr., 321, 249

Berghoefer, T. W., Schmitt, J. H. M. M., Danner, R., \& Cassinelli, J. P. 1997, A\&A, 322, 167

Bohlin, R. C., Savage, B. D., \& Drake, J. F. 1978, ApJ, 224, 132

Broos, P. S., Townsley, L. K., Feigelson, E. D., et al. 2010, ApJ, 714, 1582

Chlebowski, T., \& Garmany, C. D. 1991, ApJ, 368, 241

Clark, J. S., Egan, M. P., Crowther, P. A., et al. 2003, A\&A, 412, 185

Clark, J. S., Negueruela, I., Crowther, P. A., \& Goodwin, S. P. 2005a, A\&A, 434, 949

Clark, J. S., Larionov, V. M., \& Arkharov, A. 2005b, A\&A, 435, 239

Clark, J. S., Muno, M. P., Negueruela, I., et al. 2008, A\&A, 477, 147

Clark, J. S., Davies, B., Najarro, F., et al. 2009a, A\&A, 504, 429

Clark, J. S., Crowther, P. A., Larionov, V. M., et al. 2009b, A\&A, 507, 1555

Clark, J. S., Najarro, F., Negueruela, I., et al. 2011, A\&A, submitted

Corcoran, M. F. 2005, AJ, 129, 2018

Corcoran, M. F. 2011, Bull. Soc. Roy. Sci. Liège, 80, 578

Corcoran, M. F., Hamaguchi, K., Pittard, J. M., et al. 2010, ApJ, 725, 1528

Crowther, P. A., Lennon, D. J., \& Walborn, N. R. 2006, A\&A, 446, 279

Damineli, A. 1996, ApJ, 460, L49

Davidson, K. 1989, Physics of Luminous Blue Variables, ed. Davidson et al.

(Dordrecht and Boston: Kluwer Academic Publishers), IAU Colloq., 113, 101

Davidson, K., \& Humphreys, R. M. 1997, ARA\&A, 35, 1

Egan, M. P., Clark, J. S., Mizuno, D. R., et al. 2002, ApJ, 572, 288

Eikenberry, S. S., Matthews, K., LaVine, J. L., et al. 2004, ApJ, 616, 506

Figer, D. F., Najarro, F., \& Kudritzki, R. P. 2004, ApJ, 610, L109

Güdel, M., \& Nazé, Y. 2009, A\&ARv, 17, 309

Gvaramadze, V. V., Kniazev, A. Y., Fabrika, S., et al. 2010a, MNRAS, 405, 520

Gvaramadze, V. V., Kniazev, A. Y., \& Fabrika, S. 2010b, MNRAS, 405, 1047

Harnden, F. R. Jr., Branduardi, G., Elvis, M., et al. 1979, ApJ, 234, L51

Humphreys, R. M., \& Davidson, K. 1994, PASP, 106, 1025

Hamaguchi, K., Corcoran, M. F., Gull, T., et al. 2007, ApJ, 663, 522

Ishibashi, K., Corcoran, M. F., Davidson, K., et al. 1999, ApJ, 524, 983

Jiménez-Esteban, F. M., Rizzo, J. R., \& Palau, A. 2010, ApJ, 713, 429

Kashi, A. 2010, MNRAS, 405, 1924

Kashi, A., \& Soker, N. 2010, ApJ, 723, 602

Koenigsberger, G. 2004, Rev. Mex. Astron. Astrofis., 40, 107
Koenigsberger, G., Georgiev, L., Hillier, D. J., et al. 2010, AJ, 139, 2600

Klochkova, V. G., \& Chentsov, E. L. 2004, Astron. Rep., 48, 1005

Ku, W. H.-M., \& Chanan, G. A. 1979, ApJ, 234, L59

Lamers, H. J. G. L. M. 1989, Physics of Luminous Blue Variables, ed. Davidson et al. (Dordrecht and Boston: Kluwer Academic Publishers), IAU Colloq., 113,135

Lamers, H. J. G. L. M., Nota, A., Panagia, N., Smith, L. J., \& Langer, N. 2001, ApJ, 551, 764

Leitherer, C., Hefele, H., Stahl, O., \& Wolf, B. 1982, A\&A, 108, 102

Marcolino, W. L. F., de Araújo, F. X., Lorenz-Martins, S., \& Fernandes, M. B. 2007, AJ, 133, 489

Martins, F., Genzel, R., Hillier, D. J., et al. 2007, A\&A, 468, 233

Massey, P., \& Thompson, A. B. 1991, AJ, 101, 1408

Mauerhan, J. C., Morris, M. R., Cotera, A., et al. 2010, ApJ, 713, L33

Miroshnichenko, A. S., Bjorkman, K. S., Grosso, M., et al. 2005, MNRAS, 364 , 335

Moffat, A. F. J., Corcoran, M. F., Stevens, I. R., et al. 2002, ApJ, 573, 191

Muno, M. P., Bower, G. C., Burgasser, A. J., et al. 2006, ApJ, 638, 183

Muno, M. P., Bauer, F. E., Baganoff, F. K., et al. 2009, ApJS, 181, 110

Najarro, F. 2001, P Cygni 2000: 400 Years of Progress, ed. de Groot, \& Sterken (San Francisco: APS), ASP Conf. Proc., 233, 133

Najarro, F., Hillier, D. J., \& Stahl, O. 1997, A\&A, 326, 1117

Najarro, F., Figer, D. F., Hillier, D. J., Geballe, T. R., \& Kudritzki, R. P. 2009, ApJ, 691, 1816

Nazé, Y. 2009, A\&A, 506, 1055

Nazé, Y. 2011, Bull. Soc. Roy. Sci. Liège, 80, 109

Nazé, Y., Hartwell, J. M., Stevens, I. R., et al. 2002, ApJ, 580, 225

Nazé, Y., Manfroid, J., Stevens, I. R., Corcoran, M. F., \& Flores, A. 2004, ApJ, 608, 208

Nazé, Y., Corcoran, M. F., Koenigsberger, G., \& Moffat, A. F. J. 2007, ApJ, 658, L25

Nazé, Y., Broos, P. S., Oskinova, L., et al. 2011a, ApJS, 194, 7

Nazé, Y., Zhekov, S. A., \& Walborn, N. R. 2011b, ApJ, submitted

Oskinova, L. M. 2005a, MNRAS, 361, 679

Oskinova, L. M. 2005b, Massive Stars and High-Energy Emission in OB Associations, a workshop of the JENAM 2005 Distant Worlds, ed. Rauw et al., 99, http://www.astro.ulg.ac.be/RPub/Colloques/JENAM/ proceedings/massive/lmo.pdf

Oskinova, L. M., Ignace, R., Hamann, W.-R., Pollock, A. M. T., \& Brown, J. C. 2003, A\&A, 402, 755

Parkin, E. R., Pittard, J. M., Corcoran, M. F., \& Hamaguchi, K. 2011, ApJ, 726, 105

Pallavicini, R., Golub, L., Rosner, R., et al. 1981, ApJ, 248, 279

Pasquali, A., Comerón, F., \& Nota, A. 2006, A\&A, 448, 589

Pollock, A. M. T. 1987, ApJ, 320, 283

Rauw, G. 2011, A\&A, 536, A31

Ritchie, B. W., Clark, J. S., Negueruela, I., \& Najarro, F. 2009, A\&A, 507, 1597

Sana, H., \& Evans, C. J. 2011, IAU Symp., 272, ed. Neiner et al. (Cambridge: Cambridge University Press), 474

Sana, H., Rauw, G., Nazé, Y., Gosset, E., \& Vreux, J.-M. 2006, MNRAS, 372, 661

Seward, F. D., Forman, W. R., Giacconi, R., et al. 1979, ApJ, 234, L55

Smartt, S. J., Lennon, D. J., Kudritzki, R. P., et al. 2002, A\&A, 391, 979

Smith, N. 2008, Clumping in Hot-Star Winds, ed. Hamann et al., 27, http:// opus . kobv.de/ubp/volltexte/2008/1765/pdf/Article04.pdf

Smith, N. 2011, Bull. Soc. Roy. Sci. Liège, 80, 322

Smith, N., Li, W., Silverman, J. M., Ganeshalingam, M., \& Filippenko, A. V. 2011, MNRAS, 415, 773

Souza, S. P., \& Lutz, B. L. 1980, ApJ, 235, L87

van Genderen, A. M. 2001, A\&A, 366, 508

van Genderen, A. M., van den Bosch, F. C., Dessing, F., et al. 1992, A\&A, 264, 88

Wachter, S., Mauerhan, J. C., Van Dyk, S. D., et al. 2010, AJ, 139, 2330

Wachter, S., Mauerhan, J., van Dyk, S., Hoard, D. W., \& Morris, P. 2011, Bull. Soc. Roy. Sci. Liège, 80, 291

Walborn, N. R. 1972, AJ, 77, 312

Wang, J., \& Zhu, Z. 2003, Chinese Phys. Lett., 20, 778

Williams, P. 2011, Bull. Soc. Roy. Sci. Liège, 80, 595 